Revista Digital Año 7. Nº 9 - Año 2016. --pág. 1-104

ISSN 1853-1393

Resistencia. Chaco. Argentina - 2016

\title{
LA EXPRESIÓN CORPORAL-DANZA COMO CONSTITUYENTE DE CONCIENCIA DE CORPOREIDAD EN LA FORMACIÓN DOCENTE UNIVERSITARIA
}

\section{THE BODY-DANCE EXPRESSION AS CORPOREITY CONSTITUENT CONSCIOUSNESS AT THE UNIVERSITY TEACHER TRAINING}

\author{
Lic. Elcira Claudia Guillén ${ }^{1}$
}

Fecha de recepción: 08-08-2016

Fecha de aceptación y versión final: 11-10-2016

Resumen: La corporeidad es constitutiva del ser humano, somos "sujeto" a medida (y en la medida) en que tomamos conciencia de nuestra realidad de seres encarnados. Define nuestro límite subjetivo: la piel demarca nuestro "territorio", y al mismo tiempo conforma nuestra complexión social y cultural; la piel nos contacta con los otros para poder re-conocernos como parte de una sociedad, como integrantes de una cultura que vamos permanentemente re-creando a través de nuestra condición de seres creativos. Asimismo, nuestro cuerpo -organismo inscripto en un tiempo y un espacio- nos inmersa en la materialidad viva que integramos en un mundo multi-universal con el que compartimos la misma materia. La Expresión Corporal-Danza involucra y dimensiona sujetos encarnados y atravesados por su historia y su cultura ¿de qué modo impacta su vivencia en la formación superior universitaria? La investigación de la Facultad de Artes, Diseño y Ciencias de la Cultura titulada: "Actuar el Cuerpo. Un estudio exploratorio sobre la Expresión Corporal en la formación de alumnos del Profesorado y Licenciatura en Educación Inicial" (PI N002-2014), aprobada por Resolución № 0155/15-CS, define como uno de sus objetivos "Indagar los efectos de la Expresión Corporal en el desarrollo de una conciencia holística del cuerpo y en el proceso de constitución de subjetividades dentro de la formación docente profesional en Educación Inicial". Así, este artículo propone reflexionar sobre el lugar que tiene en la formación de los y las estudiantes universitarios, la constitución de su subjetividad.

\section{Palabras clave: Conciencia de corporeidad; Expresión Corporal-Danza; Formación superior}

Abstract: Corporeity is constitutive of the human being, we are "subject" to measure (and to the extent) that we become aware of our reality of incarnated beings. Define our subjective limit: the skin demarcates our "territory", and at the same time conforms our social and cultural complexion; The skin contacts us with others in order to re-know ourselves as part of a society, as members of a culture that we are constantly re-creating through our status as creative beings. Likewise, our body - an organism inscribed in a time and space - immerses us in the living materiality that we integrate into a multi-universal world with which we share the same matter. The Body-Dance Expression involves and dimension subjects incarnated and crossed by their history and their culture, in what way does their experience in higher university formation impact? The research of the Faculty of Arts, Design and Cultural Sciences entitled: "Acting the Body. An exploratory study on Body Expression in the training of teachers and undergraduate students in Initial Education "(PI N002-2014), approved by Resolution No. 0155/15-CS, defines one of its objectives" To investigate the effects of Body Expression in the development of a holistic consciousness of the body and

\footnotetext{
${ }^{1}$ Secretara Académica y Docente titular por concurso en la asignatura Metodología de la Investigación Artística de la Licenciatura en Artes Combinadas de la Facultad de Ates Diseño y Ciencias de la Cultura; y Docente titular por concurso en la asignatura Taller de Expresión Corporal en el Departamento de Educación Inicial de la Facultad de Humanidades, ambas Unidades Académicas de la Universidad Nacional del Nordeste, en Resistencia, CHACO, Argentina. Domicilio: Los Aromos 3815, Resistencia, Chaco, Argentina. Teléfono: 054-362-15-466-9290. Correos electrónicos: cguillen_aryahoo.com / secacad@artes.unne.edu.ar
} 
in the process of constitution of subjectivities within the professional teacher training in Initial Education ". Then, this article proposes to reflect on the place that has in the training of university students, the constitution of their subjectivity.

Key words: Corporeity, Expression Corporal-Danza, y higher education

\section{Introducción:}

Vivimos una concepción de "cuerpo" devenida de la perspectiva hedonista de los antiguos griegos quienes abogaban y trabajaban por conseguir su perfección. Lo concebían en vinculación con el arte, y a éste como máxima expresión a la que pueda llegar el ser humano.

"Su concepto de arte se apoyaba en la síntesis de lo bueno y lo bello, lo que implica una conexión con lo mental, lo moral y lo físico, pugnando por un desarrollo interior a partir del cultivo de la apariencia externa. De ahí que se abandere el eslogan de: 'mente sana en cuerpo sano'. Presuponiendo que la salud en su amplio sentido solo puede ser albergado en un cuerpo bien cuidado, estético y bello". (Alfonso Espinal, 2006).

Impuesta por Platón, la cultura occidental plantea desde sus orígenes una visión dual cuerpo-mente, y la dicotomía cuerpo-espíritu implantada por San Agustín desde la reflexión teológica sostenida durante siglos, agudizó esta perspectiva. Las ciencias también acuerdan duplicidades corporales: por un lado existe una entidad visible y palpable que es comprobable científicamente en sus múltiples funcionalidades; y por el otro una naturaleza invisible e intangible que se concibe como reducto de la filosofía y las religiones. Como consecuencia. Vivimos en un cuerpo habitado por un "inquilino" alojado temporalmente en él; un cuerpo a merced de la mente todopoderosa. Estas son ideas que se relacionan muy bien con la concepción de "cuerpo producto" que nos lleva a percibirnos como "cuerpos dislocados" (Grasso, 2012, p. 19).

Haciendo un breve recorrido por las últimas décadas de la cultura eurocentrista, es posible reconocer mutaciones en cuanto a la concepción del cuerpo, aunque siempre dentro de esta visión dualista en relación a la constitución de subjetividad. En los sesenta sobrevino la "revolución sexual" interrumpiendo un largo período durante el que el cuerpo y sus impulsos estuvieron doblegados a la mente y al espíritu. Así, un nuevo imaginario de cuerpo "lujurioso" irrumpió en la sociedad invadiendo prácticamente todas las parcelas de las prácticas sociales, "convirtiendo al cuerpo en un signo de reunión, un caballito de batalla contra un sistema de valores considerado represivo y perimido que era necesario transformar para garantizar las libertades individuales" (Le Breton, 2002, p. 10). Surgieron entonces, prácticas y discursos sociales que propusieron o exigieron una transformación radical de los antiguos marcos sociales. Se invitaba a la "liberación del cuerpo", discurso en el que éste, está planteado como una posesión, como un atributo del hombre y la mujer utilizado para expresar insubordinación: "La apología del cuerpo es, a pesar suyo, profundamente dualista, en tanto opone al individuo y a su cuerpo." (Le Breton, 2002, p. 10). 
La década del ochenta irrumpe con un fuerte "culto" a su cuidado: una alimentación balanceada y una fuerte actividad física se instalan como sinónimos de de vida plena y feliz. Así, "el modelo social global se centra en una determinada apariencia física: el aspecto impuesto es el de un cuerpo joven, delgado, musculoso y limpio." (Grasso, 2012, p. 19).

Los 90 inician décadas en las que se imponen métodos más o menos agresivos, justificados por acercarse a la apariencia física impuesta por los medios y vinculada al éxito. "Entonces, el cuerpo se convierte en un campo de batalla, en una lucha cuya finalidad es su aceptación ante nuestra mirada y la del otro. Identidad y pertenencia pasan por la apariencia" (Grasso, 2012, p. 20).

Mens sana in corpore sano es una máxima que ha circulado fuertemente durante todo el siglo XX, y las fronteras del siglo XXI han heredado sus conceptos descendientes. La dicotomía presente en esta sentencia, ha prevalecido durante décadas en nuestros sistemas educativos de todos los niveles, creando compartimentos estancos; la mente puede dominar el cuerpo, y este se somete a través de las disciplinas físicas. En este binomio es imposible alcanzar una armonía, "se parte de principio dicotómico de 'habilidades del pensamiento' por un lado y 'habilidades motrices' por otro, a los que se sumarian las 'habilidades sociales'” (Kemelmajer, 2001, p. 23).

Simultáneamente a esto, Maurice Merlau-Ponty (1984), considerado un referente de la fenomenologíai, en la introducción de su libro Fenomenología de la Percepción, habla de la conciencia de corporeidad y la explica con estas palabras: "Percibir es tornar presente cualquier cosa con la ayuda del cuerpo. Yo no estoy delante de mi cuerpo, estoy en mi cuerpo; o mejor soy mi cuerpo" (Merlau-Ponty, 1984, p. 8). Estas expresiones proponen un significado muy diferente sobre el cuerpo al incluir la noción de corporeidad, entendida ésta "como realidad concreta mediante la cual el hombre habita el mundo" (Herrera-Espinoza, 1965 como se citó en Kemelmajer, 2001, p. 8). Así, "Corporeidad y motricidad no son simples distinciones utilizadas para refinar el lenguaje, son dos conceptos de tradición fenomenológica que han empezado a ser utilizados en los últimos tiempos" (Hurtado Herrera, 2008, P. 119).

\section{Conciencia de Corporeidad y Constitución del Ser Humano:}

El concepto que entiende la corporeidad como una compleja trama en la que se entrecruzan diferentes dimensiones constitutivas de la identidad de cada persona, la describe como "la vivenciación del hacer, sentir, pensar y querer" (Zubiri, 1986 como se citó en Paredes, 2003), donde el cuerpo es sólo el vehículo para que ésta se haga presente en el mundo y para el mundo que lo rodea. Es decir, el cuerpo es el medio para que se manifieste y desarrolle la corporeidad y se constituya la subjetividad: el sujeto encarnado. (Alfonso Espinal, 2006). Así, escuchar lo que el cuerpo dice es integrar esta idea de corporeidad como construcción y desarrollo de conciencia corporal.

Dice Jesús Paredes (2003): "El ser humano se expresa, se comunica, vive con, por y a través de su corporeidad. Podríamos decir que la epifanía del ser humano es su cuerpo y a partir de él desarrollamos la corporeidad (aspecto físico, psíquico y anímico)". Eugenia Trigo (1999), la define como expresión de lo humano, considerando que la persona se manifiesta a través y con su cuerpo, y son esas 
manifestaciones -emociones, sentimientos, pensamientos- las que se corporeizan. Por esto, hablar del cuerpo humano en toda su amplitud, es transcender el sistema orgánico para entender y comprender su todo yo encarnado. Dicho de otra manera, es concebir al ser humano con conciencia holística: considerar que el "todo" es un sistema más complejo que una simple suma de sus elementos constituyentes, y que su naturaleza como ente no es derivable de sus elementos constituyentes. Así se concibe al sujeto (que es en su cuerpo) comprendiendo el sinergismo entre las partes y no la individualidad de cada una, ya que "La corporeidad se construye con la suma y la integración de los cuerpos vividos”. (Grasso, 2012, p. 43)

Alfonso Espinal (2006), agrega un elemento más a esta concepción, entiende la corporeidad como la manifestación consciente o inconsciente de la constitución física del individuo, moldeada y reclamada en un determinado contexto, definiéndose en el encuentro de nuestros los físicos (de una estructura biológica capaz de moverse), con los mentales (razón, emoción y pensamiento) y los sociales (ánimo, voluntad y motivación). Siguiendo esta concepción, la triada de cuerpo, mente y socialización que conforma a la corporeidad, es lo que define al individuo como ser humano, siendo la cultura indudablemente parte constitutiva de él. "En este enfoque, el hombre es concebido como la emanación de un medio social y cultural." (Le Beton 2002, p.16), así es como clasificamos y atribuimos valor a los innumerables estímulos que recibimos de nuestro cuerpo a través de las actividades perceptivas y desde la palabra, pero también desde los gestos y ademanes, y así es como establecemos ese intercambio que nos define como individuos insertos, pertenecientes y constructores de un colectivo. "Corporeidad y encarnación son conceptos que reconocen los procesos mediante los cuales, la cultura se inscribe en el cuerpo, procesos que nos permiten encontrar en él un espacio de convergencia discursiva, un lugar de intervención" (Hurtado Herrera, 2008, p. 122)

Nuestro cuerpo existe en la totalidad de sus componentes con la historia (experiencias) con la que ha sido atravesado, la educación sistematizada es uno de los mayores modos de asimilación del medioambiente. Aprendemos a comportarnos socialmente con el cuerpo, y este aprendizaje continúa durante toda nuestra vida. En esta trama, "Los otros contribuyen a dibujar los contornos de su universo y a darle al cuerpo el relieve social que necesita, le ofrecen la posibilidad de construirse como actor a tiempo completo de la colectividad a la que pertenece" (Le Breton, 2002, p. 9), de aquí la importancia que consideramos tiene, la conciencia de corporeidad, a la que se entiende como el comprender que lo que somos como sujetos es resultado de la historia que encarnamos, y no sólo de lo que sabemos, creemos o pensamos.

Si nos remontamos a las primeras décadas del siglo $X X$, encontramos el momento en el que el paradigma evolucionista impuso la idea de que la inteligencia tiene una base biológica y que cumple una función fundamental en el logro de la supervivencia. La biología molecular por su parte, confirma un aspecto de este paradigma aunque refuta la idea de la evolución de la inteligencia humana. Por otro lado, la sociología, la antropología y la historia mostraron que la conciencia -si bien dimensión ontológica del ser humano- evoluciona en función de la experiencia social. Es decir, el saber sobre nosotros mismos y sobre lo que sabemos es un descubrimiento relativamente reciente. 
Por esto, ahora no se habla de inteligencia sino de proceso del conocimiento y se intenta determinar cómo interactúan el mundo externo, la subjetividad, el lenguaje, las ideas, el arte y la producción artística, y las experiencias sociales en la constitución del sujeto que encarna ese proceso. Una respuesta consensuada plantea entender que el carácter del conocimiento es transdisciplinario, así como el de la inteligencia humana.

Si bien en el mundo hay coincidencia en concebir al conocimiento como la norma del comportamiento humano, los herederos de la cultura eurocentrista en general, tenemos una larga trayectoria en entender la inteligencia como el medio para comprender la realidad del hombre a partir de concebir a la razón como una parte del mundo natural: el platonismo y el aristotelismo nos plantearon "la realidad" como paradigmas contrapuestos. En oriente en cambio, se entiende que la conciencia está por encima de todo, no como sustancia ni determinada por sustancia natural alguna, lo que permite afirmar la trascendencia de la mente y de la conciencia humana independientemente de concepciones científicas y religiosas.

Edgar Morin (1999) concibe la corporeidad como condición humana, y sostiene que la interrogación sobre ella permite el cuestionamiento sobre la situación del ser humano en el mundo. Plantea que durante las décadas del 1960 y 1970 se han desarrollado teorías sobre cosmología, ecología, biología, prehistoria, que han modificado las ideas modernistas sobre el universo, el planeta tierra, la vida y el mismo ser humano, aunque por estar estos aportes recluidos en diferentes ciencias y disciplinas, lo perteneciente a lo humano se halla fragmentado, produciéndose un problema epistemológico.

Lo Humano permanece cruelmente dividido, fragmentado en pedazos de un rompecabezas que perdió su figura. Aquí se enuncia un problema epistemológico: es imposible concebir la unidad compleja de lo humano por medio del pensamiento disyuntivo que concibe nuestra humanidad de manera insular por fuera del cosmos que lo rodea, de la materia física y del espíritu del cual estamos constituidos, ni tampoco por medio del pensamiento reductor que reduce la unidad humana a un substrato puramente bio-anatómico. Las mismas ciencias humanas están divididas y compartimentadas. La complejidad humana se vuelve así invisible y el hombre se desvanece 'como una huella en la arena'. Además, el nuevo saber, por no estar religado, tampoco está asimilado ni integrado. Paradojicamente, hay un agravamiento de la ignorancia del todo mientras que hay una progresión del conocimiento de las partes. (Morin, 1999, p.23).

Así, en nuestra cultura y en nuestras universidades, se plantea una barrera entre el desarrollo del conocimiento y el desarrollo de la conciencia al concebir que la mente tenga el poder para descifrar las leyes de la naturaleza y crear nuevas condiciones de vida, atribuyéndole a ella, y sólo a ella, la constitución de una humanidad armoniosa. No se incluyen en la formación académica aquellos aspectos del individuo que lo constituyen en su realidad trascendental; ni se lo comprende como sujeto vehiculizado por una realidad social encarnada en su cuerpo. Este concepto en el desarrollo de la ciencia no se ha tenido en cuenta.

Se observa que en la mayoría de los casos, la formación superior universitaria (la no universitaria también, pero hacemos un recorte en la universitaria) deja de lado al estudiante/sujeto que encarna una cultura atravesada por una historia 
personal/social. Aparentemente aún no se asume en los hechos que "La posibilidad del conocimiento supone la conciencia y la libertad, del mismo modo que supone el cerebro y la dotación genética del ser humano" (Pérez Lindo, s. f.).

Con todo esto ¿será posible sostener que la conciencia de corporeidad mediatiza la libertad del sujeto y de las sociedades? La hipótesis que proponemos en este artículo dice que sí, que brinda autonomía y autoafirmación, y concibe individuos emancipados en y a través de las sociedades. Brindarle a los sujetos el espaciotiempo para la reflexión (entendiéndola como resultado de la meditación y de la posibilidad de flexionar sobre sí mismo), posibilita la metacognición: la capacidad de anticipar la conducta (propia y ajena) a partir de percibir emociones y sentimientos es posible cuando se "enseña" y se "admite" percibirse y actuar en consecuencia de ello. Es un hecho que en nuestras escuelas y universidades escasea esta práctica.

Edgar Morin (1999), aporta en esta disquisición cuando dice:

"La educación del futuro deberá ser una enseñanza primera y universal centrada en la condición humana. Estamos en la era planetaria; una aventura común se apodera de los humanos donde quiera que estén. Estos deben reconocerse en su humanidad común y, al mismo tiempo, reconocer la diversidad cultural inherente a todo cuanto es humano. Conocer lo humano es, principalmente, situarlo en el universo y a la vez separarlo de él". (Morin, 1999, p. 23)

Tomando como punto de partida el breve análisis realizado en la introducción de este artículo, otorgando validez a lo expuesto en el punto definido como Conciencia de Corporeidad y Constitución del Ser Humano, y entendiendo el papel fundamental que juega la educación como posibilitadora (o no) del puente entre saber que tenemos un cuerpo y tomar conciencia de que somos en él, se propone a continuación, el desarrollo de un nuevo título para hablar de los atributos disciplinares de la Expresión Corporal-Danza como facilitadora del desarrollo de conciencia de corporeidad -o conciencia holística- en los sujetos y sociedades.

\section{Sobre la Expresión Corporal-Danza:}

El espacio-tiempo entre y el momento posterior a las dos grandes Guerras Mundiales, establecieron casi dos décadas emblemáticas en la cultura occidental. Época de roturas y quiebres que expresados en todos los lenguajes del arte, propusieron las bases para nuevos paradigmas y búsquedas, fundamentalmente se planteó la ruptura de las "formas".

La danza introdujo temáticas diferentes a las de seres supra-terrenales y comenzó a bailar sin las zapatillas de puntas. Emprendió un camino de búsqueda encarnada en cuerpos semi desnudos y con el pelo suelto. Su musa inspiradora en Estados Unidos, fue Isadora Duncan. En Europa, Rudolf Von Laban investigó las bases del movimiento orgánico y definió los elementos del movimiento dancístico creando un nuevo concepto de danza al que llamo free dance. Moshe Feldenkrais (refugiado del nazismo en Inglaterra), propuso una técnica en la búsqueda del movimiento consciente. Jacques Dalcroze investigó la internalización de la música a través del movimiento y creo un método para su enseñanza, la "euritmia". Ellos fueron maestros de Patricia Stokoe y sin saberlo, establecieron las bases de la estructura disciplinar de lo que hoy conocemos en la Argentina, como Expresión Corporal-Danza. 
Patricia Stokoe -argentina hija de ingleses- en el año 1938 viaja con su familia a la convulsionada Europa que estaba gestando la segunda guerra mundial. Ella permanece en Inglaterra con el objetivo de formarse y regresa doce años más tarde. Su máximo propósito: introducir este nuevo concepto de danza en la argentina de la década del 50 apostando a dejar de lado posibles prejuicios sobre lo que la danza podía implicar.

\begin{abstract}
“(...) reemplacé 'Danza Libre (traducción al castellano de "Free Dance', de Rudolf von Laban, que utilicé cuando empecé a trabajar en Buenos Aires) por 'Expresión Corporal', sin agregar la palabra Danza al final (...) Pasaron cuarenta años y la falta de esa palabra, 'danza', contribuyó a una confusión (...) Hace ya algunos años me di cuenta de lo que había sucedido y volví a agregar la palabra 'danza' al rótulo, no para cambiar el significado de mi trabajo, sino para aclararlo. 'Expresión Corporal Danza' significa 'tu danza'”. (Stokoe, 1992, p. 34)
\end{abstract}

Esta concepción, configura al sujeto que transita la construcción de este lenguaje corporal expresivo, como coreógrafo e intérprete de sí mismo, concibiendo que esto es posible sólo cuando tomamos conciencia de que "somos en un cuerpo", y el movimiento expresa nuestra realidad espacial, temporal y energética.

Patricia Stokoe desarrolla una técnica -columna vertebral de esta disciplina artística- a la que denomina Sensopercepción. Ésta, mediatiza la posibilidad de conexión con el mundo interior de cada sujeto al mismo tiempo que lo dimensiona como individuo atravesado por la cultura y la historia de vida. Al poner el cuerpo en movimiento desde el propio eje, se baila desde el centro de gravedad conectando el mundo interior: desde la propioceptividad hacia el movimiento expresivo.

Así, la Expresión Corporal nace estableciendo un nuevo paradigma en el concepto de danza al recuperar la danza como lenguaje del hombre y la mujer. Roger Garaudy en su libro "Danzar su vida" (2003) enfatiza de una manera sumamente poética el aspecto trascendental que el hombre conecta de sí a través de la nueva concepción de danza, Dice: "Danzar es ante todo, establecer una relación activa entre el hombre y la naturaleza y participar del movimiento cósmico y del dominio sobre él (...) La danza vuelve a Dios presente y al hombre potente, es la presencia del espíritu en la carne". (Garaudy, 2003, p. 14). El describir esta génesis profunda de la danza, nos hace tomar conciencia de su significado como símbolo del acto de vivir y como fuente de toda cultura, porque desde el origen de las sociedades por las danzas y por los cantos el hombre se afirma como miembro de una comunidad que lo trasciende, ya que esta forma de danza mediatiza su constitución como sujeto social. El "bailarse" potencia en el sujeto su conciencia holística.

En su naturaleza disciplinar, la Expresión Corporal-Danza define como su campo de conocimiento aspectos que son constitutivos del ser humano: La Corporeidad; la Espacialidad; la Temporalidad; la Comunicación; y la Creatividad. Es por esto que el vivenciarla confronta nuestra esencia y fortalece nuestras posibilidades. Nuestra esencia se dimensiona en nuestra realidad de seres corpóreos; creativos; dimensionados en un espacio-tiempo; definidos en una sociedad a la que definimos y en la que desplegamos cultura y ciudadanía. Nuestra potencia radica en expresar creativamente nuestro mundo interior y generar productos innovadores (ciencia, tecnología, arte); algunos de los modos para hacerlo son los lenguajes artísticoexpresivos, uno de ellos es la danza. 


\title{
El ejercicio de la Expresión Corporal en la formación profesional docente:
}

\section{En nuestras universidades, prevalece}

"una concepción biologisista y pragmática del hombre (y por lo tanto del cuerpo); al conceptualizarlo así, se espera como un instrumento útil, eficiente y productivo, lo que deviene en la ideología de una sociedad capitalista (...) Se habla entonces de un cuerpo sometido, dominado y controlado por un sistema social que le dicta cómo debe ser". (Espinal, 2006)

Si bien las Universidades argentinas se plantean reformular las concepciones de enseñanza a partir de la necesidad de producir investigaciones innovadoras y acciones que las vinculen con el medio social y económico, no les es posible aún definir ni aplicar procedimientos que generen una ruptura en esa concepción "disciplinista" y dicotómica con la que abordan la trasmisión de conocimientos. Esto preocupa más en las facultades que se ocupan de las ciencias humanas y de la cultura.

Ocurre que en la formación superior universitaria, no se tiene en cuenta que "La reflexión corporal del ser humano pronuncia su primera manifestación usando el cuerpo, creando corporeidad de muchas maneras: desde que está en el vientre de la madre tiene necesitad de moverse; desde recién nacido siente la necesidad de expresarse y su corporeidad es su primera vía de expresión y comunicación con el mundo". (Paredes, 2003).

Se considera pertinente incluir aquí la extracción de un párrafo de "Eupalinos o el arquitecto", de Paul Valery (como fue citado por Roberto Calabria ii, 1992, p. 45)

\begin{abstract}
"Ese cuerpo es un Instrumento admirable, de que los vivientes, que le tienen todos a su servicio, no usan, según me cercioré, en su plenitud, no sacan de él sino placer y dolor y actos indispensables, como el vivir. Ya con él se confunden, ya por algún Lapso olvidan su existencia; y ya brutos, ya espíritus puros ignoran los vínculos universales que contienen y de qué sustancia prodigiosa fueron hechos".
\end{abstract}

Este texto le permite a Calabria (1992) reflexionar de la siguiente manera:

"Su verdad vale incondicionalmente y más para el hombre teórico que, idealista o materialista, tiende a olvidar la existencia del cuerpo (de su cuerpo) por 'algunos lapsos'. Una educación que se remita al cuerpo según esos principios (tal la que propone Bayerthal) tendrá un campo de aplicabilidad tan amplio como los vínculos universales lo permitan. Ahora bien ¿cómo procesar esa remisión?" (Calabria, 1992, p. 45)

Así como Calabria (1992) propone las técnicas y metodología desarrollada por Bayerthal en la gimnasia consciente, yo propongo la Expresión Corporal-Danza como disciplina, que en su ejercicio, viabiliza en las personas los mecanismos capaces de desarrollo de conciencia corpórea, entendiéndola como aquello que nos instituye en nuestra humanidad. Y aquí resulta necesario hablar un poco más de la Expresión Corporal-Danza.

Esta disciplina artística inscribe en su esencia, que en la realidad corporal de los sujetos que la experimentan en la búsqueda y el encuentro de la propia danza, está contenido el mensaje y el canal que lo concretiza y comunica. A través de la 
Sensopercepción, "pretende recuperar y enriquecer la vivencia del propio cuerpo para la danza, para la vida. Es una práctica de descubrimiento y despliegue de nuestro potencial con una fundamentación teórica que puede ser abordada desde diversos campos". (Kalmar, D; Gubbay, M., 2012 iii).

Así, la Expresión Corporal-Danza que además -y como ya se dijo- sistematiza en su campo de conocimiento aspectos constitutivos de la condición humana, es una propuesta invaluable de práctica capaz de transformar la dicotomía que impera en la cultura y en las aulas universitarias. Es necesario para ello, entre otras cosas, promover investigaciones que denuncien las distancias entre los propósitos de las universidades (producir conocimiento al servicio de la emancipación de los sujetos, las culturas y las sociedades) y su abordaje segmentado en la trasmisión de conocimiento, por un lado, y por otro y al mismo tiempo, plantear alternativas que den respuesta a estas problemáticas.

Para que algo sea aprendido debe haber atravesado el cuerpo, ya que no existe aprendizaje fuera de la experiencia. Porque como lo describe Edgar Morin (1999, p. 16): "Las unidades complejas, como el ser humano o la sociedad, son multidimensionales; el ser humano es a la vez biológico, síquico, social, afectivo, racional. La sociedad comporta dimensiones históricas, económicas, sociológicas, religiosas... El conocimiento pertinente debe reconocer esta multídimensíonalidad e insertar allí sus informaciones".

Las Universidades son sin dudas, unidades complejasiv, por ello se concibe como Morin que:

"El conocimiento pertinente debe enfrentar la complejidad. Complexus significa lo que está tejido junto; en efecto, hay complejidad cuando son inseparables los elementos diferentes que constituyen un todo (como el económico, el político, el sociológico, el sicológico, el afectivo, el mitológico) y que existe un tejido interdependiente, interactivo e inter-retroactivo entre el objeto de conocimiento y su contexto, las partes y el todo, el todo y las partes, las partes entre ellas. Por esto, la complejidad es la unión entre la unidad y la multiplicidad." (Morin, 1999, p. 17).

Siendo consecuentes con esta idea, el proyecto de investigación denominado Actuar el Cuerpo. Un estudio exploratorio sobre la Expresión Corporal en la formación de alumnos del Profesorado y Licenciatura en Educación Inicial, vincula las áreas temáticas de la formación docente y el ejercicio artístico. Su propósito es medir el alcance que tiene la práctica de la Expresión Corporal-Danza en la formación profesional y el desarrollo personal (cuestiones que se consideran sincrónicas) en las estudiantes del profesorado y licenciatura en Educación Inicial. Uno de sus objetivos plantea: Indagar los efectos de la Expresión Corporal en el desarrollo de una conciencia holística del cuerpo (corporeidad) y en el proceso de constitución de subjetividades dentro de la formación docente profesional en Educación Inicial.

Esta investigación, si bien se desarrolla en la Facultad de Artes, Diseño y Ciencias de la cultura (FADyCC) de la Universidad Nacional del Nordeste (UNNE), recoge experiencias realizadas por alumnas que cursan la cátedra Taller de Expresión Corporal del Profesorado y Licenciatura en Educación Inicial del Departamento de Educación Inicial de la Facultad de Humanidades de la misma universidad. Los resultados obtenidos en proyectos académicos desarrollados consecutivamente desde el año 2012, que inter-vinculan el enfoque definido en la asignatura "Taller de Expresión Corporal" (Área de las disciplinas especiales) con el de la asignatura "Taller 
de Integración, Investigación y Práctica III" (Área de las Prácticas Profesionales), han producido trabajos presentados en Jornadas y Congresos Nacionales e Internacionales de Educación y de Educación Artística, que han podido dimensionar la importancia del ejercicio de la Expresión Corporal-Danza en las estudiantes que se forman como profesionales en la educación inicial.

A lo largo de cinco años se han puesto en práctica la vinculación de una disciplina artística con las prácticas docentes en instituciones del nivel inicial en proyectos inter-cátedra. Así fue posible comprender la importancia que tiene la conciencia de corporeidad en la formación profesional docente. Si ésta no es incluida en la formación de estas futuras formadoras, no es viable esperar que tengan la posibilidad de realizar quiebres en las prácticas establecidas, en lo instituido. "Brian Fay argumenta que el aprendizaje no es un simple proceso cognitivo, sino también un proceso somático en los que la opresión deja sus huellas no solo en las mentes de las personas, sino también en sus músculos y en sus esqueletos" (Braian Fay, 1987,p. 146, como se citó en Mc Laren, 1997, pp. 88, 89)

Se concibe que la Expresión Corporal en tanto disciplina artística que se instituye y concretiza en el movimiento dancístico, provee de un recurso invaluable en el autoconocimiento del cuerpo, y por lo tanto del sujeto encarnado en él, es decir en la constitución de subjetividad. Por tanto, esta experiencia puede incidir positivamente en la formación profesional del docente en particular -en este caso en Educación Inicial-, y de manera general, en la formación artística y profesional, aún en aquellas disciplinas denominadas "ciencias duras" y en las "formaciones independientes". Las universidades forman personas que serán profesionales que se insertarán en sus comunidades.

Tuve el privilegio de haber sido alumna de Patricia Stokoe, esa gran persona que refiriéndose a ella misma y a lo que definió como su hacer decía:

"Trabajo la corriente de Expresión Corporal que la considera Danza, aquella que desarrolla las características personales y por eso accesibles y dentro del alcance de cada ser humano, que será su danza, la que él puede manejar, la danza pensada como producto único, la poesía corporal de cada individuo (...) La poesía está en cada ser humano, en todos, no solo en los grandes. De la misma manera pensamos que la danza está en todos, no solamente en los bailarines profesionales." (Patricia Stokoe en Kalmar y Gubbay, 2012, p. 10)

\section{Discusión}

La Facultad de Artes, Diseño y Ciencias de la Cultura ha sido creada en la UNNE hace tan sólo un lustro, al inicio de la segunda década del siglo XXI, y si bien sus carreras son muy jóvenes (una de ellas aún no cumple un año de vida) lleva sobre sus espaldas la posibilidad de romper viejos paradigmas instalados en las aulas universitarias. En el mundo de hoy invadido por nuevas y masivas tecnologías que han acercado las fronteras, esto es una demanda. El arte seguirá siendo aquello que expresa las latencias sociales, culturales y políticas que constituyen las sociedades. Los lenguajes artísticos y los artistas que los encarnan, han tenido a lo largo de la historia occidental, la contingencia de expresar las rupturas de los paradigmas que encapsulan, dogmatizando sobre ciencia y conocimiento. Por esto, la investigación artística propone nuevos modos de ver y vivir el mundo. 
Así, la investigación de la que se desprende este artículo, apunta a acercar dos entidades hasta ahora vividas separadamente: la formación profesional y la experiencia artística. $\mathrm{Y}$ tomando en consideración las reflexiones realizadas en esta publicación, cabe concebir que el ejercicio artístico se erige de modo contundente como camino en el alcance de uno de los propósitos de nuestras universidades: generar "estrategias que orienten las actividades educativas y científicas a la resolución de problemas de la sociedad, el estado y la economía" (Pérez Lindo, s. f.).

Resulta necesario, siguiendo a Morín (1999), interrogar al mundo concibiendo su complejidad multidimensional, y esto será posible sólo desde la conciencia de que somos seres multidimensionales, conectados y a la vez separados de él.

\section{Referencias bibliográficas}

Calabria, Roberto (1992) "Bayerthal: El Cuerpo Vivido". En Topía Revista. Psicoanálisis, Sociedad y Cultura. Año II, N. 6, Noviembre 1992, 44-49. <https://www.topia.com.ar/revista/\%C3\%A9tica-psicoan\%C3\%A1lisis-y-sociedad> [Consulta: agosto 2016]

Espinal, Alfonso (2006) "La sociología del cuerpo". Portal: psicopedagogía.com, Psicología de la educación para padres y profesionales.

<http://www.psicopedagogia.com/sociologia-cuerpo> [Consulta: septiembre

Garaudy, Roger (2003) Danzar su Vida. Chile, Editorial Consejo Nacional para la Cultura y el Arte.

Grasso, Alicia (2012) Arte y Corporeidad: una propuesta integradora. Buenos Aires, Ministerio del Río de la Plata, LUMEN.

Hurtado Herrera, D. R. (2008) "Corporeidad y motricidad. Una forma de mirar los saberes del cuerpo". Revista Educ. Soc., Campinas, vol. 29, n. 102, jan./abr, 119-136. $<$ http://www.cedes.unicamp.br/> [Consulta: agosto 2016]

Kalmar, D; Gubbay, M. (2012) "Sensopercepción". Artículo

$<$ http://es.scribd.com/doc/89255555/La-danza-debe-estar-al-alcance-de-

todos-Patricia-Stokoe> [Consulta: marzo 2012]

Kemelmajer, Jovita (2001) Movimiento Expresivo y Creatividad. Mendoza, Facultad de Educación Elemental y Especial, UNCUYO. Visión.

Le Breton, D. (2002) La Sociología del Cuerpo. Buenos Aires, Ediciones Nueva

Mc Laren, E. (1997) Pedagogía Crítica y cultura depredadora. España, Paidós.

Merleau-Ponty, M. (1984) Fenomenología de la Percepción. España, Planeta Agostini.

Morin, E. (1999) Los siete saberes necesarios para la educación del futuro. Traducción de Mercedes Vallejo-Gómez. París, Francia, UNESCO para la Educación, la Ciencia y la Cultura.

Obeide, S. (2000) "Mecanismos de Asignación de Recursos a las Universidades Nacionales: Una Perspectiva Crítica del Caso Argentino (1992-1999)". Revista Administración Pública y Sociedad, Número 13, IIFAP-UNC, 2000. [Consulta: julio 2016]

Paredes, J. (2003) Desde la corporeidad a la cultura. Revista digital Efdeportes No. 62. Julio, Buenos Aires, Argentina. [Consulta: septiembre 2016]

Pérez Lindo (s. f.). "De la revolución cognitiva a la gestión del conocimiento". Documento Base de Gestión Universitaria, Argentina, Universidad Nacional del Nordeste. [Consulta: agosto 2007]

Stokoe, Patricia (1993) “Expresión Corporal Danza: Ese Lenguaje Silenciado”. Topía Revista. Psicoanálisis, Sociedad y Cultura. Año III, N 8, agosto 1993, 33. 
<https://www.topia.com.ar/revista/\%C3\%A9tica-psicoan\%C3\%A1lisis-y-sociedad> [Consulta: marzo 2012]

Trigo, Eugenia (1999) "La corporeidad como expresión de lo humano". I Congreso Internacional de Motricidade Humana. Almada-Portugal 1999. 2014]

$<$ http://www.portalfitness.com.ar/Nota.aspx?i=7226\&p=1> [Consulta: abril

\title{
Notas
}

\begin{abstract}
${ }^{i}$ Corriente del pensamiento filosófico que surge en la década del cuarenta del siglo pasado que se basa en el estudio de los fenómenos sociales y naturales.

ii Roberto Calabria es filósofo y alumno de Inx Beyerthal, creadora de gimnasia consiente y del instituto que lleva su nombre en la ciudad de Montevideo.

iii Artículo elaborado para el profesorado del Estudio Patricia Stokoe y publicado en la Revista Uno Mismo (Bs. As., 1985) y presentado en el "Primer Encuentro Latinoamericano de Enseñanza Artística", organizado por el Ministerio de Cultura de Cuba, La Habana, Cuba, 1986.

iv "La estructura organizacional universitaria es invariablemente isomórfica a las distintas profesiones y disciplinas. Las facultades, escuelas, departamentos académicos, institutos, se crean en función del ordenamiento profesional y científico, generando un conjunto de unidades organizativas (académicas) cuya relación es inevitablemente laxa o inexistente.

"(...) A la complejidad derivada de estos aspectos, se adiciona la gran heterogeneidad de intereses formalmente representados: estudiantes, docentes, no docentes, egresados (...)." (Obeide, 2000, p. 4).
\end{abstract}

